\title{
Esquizofrenia y sustancias psicotropas de consumo frecuente en nuestro medio.
}

\author{
Santamarina, S.; Iglesias, C. \\ Servicio de Psiquiatría Hospital Valle del Nalón. Servicios de Salud Mental del Principado de Asturias.
}

Dra. Santamarina Montila. Centro de Salud Mental Valle del Nalón. C/ José Jove y Canella 1 (fundación ADARO). Langreo 33900. ASTURIAS. Tfno. 985675300

\section{RESUMEN}

El presente estudio trata de evaluar la prevalencia de los distintos trastornos por uso de algunas sustancias de uso común en nuestro medio, en una muestra de pacientes esquizofrénicos tratados y estudiar la presencia de perfiles sociodemográficos y clínicos asociados al abuso de sustancias.

Para ello se estudiaron 72 pacientes con esquizofrenia de los cuales se recogieron variables sociodemográficas y clínicas, siendo evaluados mediante el cuestionario de detección de tóxicos de la OMS, la escala del síndrome positivo y negativo (PANSS).

Durante el año previo 27 pacientes (37,5\%) presentaron consumos regulares con patrón desadaptativo y el 54,2\% (39 pacientes) cumplían criterios diagnósticos de trastorno por uso de sustancias actual o en el pasado. No se observaron asociaciones significativas para la mayoría de las variables estudiadas. El sexo masculino, estar en situación de desempleo, haber tenido mas problemas judiciales y la baja adherencia a la medicación fueron las únicas características que diferenciaron al grupo de pacientes con un diagnóstico comórbido.

Palabras clave: Esquizofrenia, abuso de sustancias, diagnostico dual.

\section{INTRODUCCIÓN}

E I término "diagnóstico dual" se emplea para describir la combinación en el mismo paciente de un trastorno mental grave (generalmente psicosis) y un trastorno por uso de sustancias. El trastorno dual tiene características clínicas propias y diferentes a las presentadas aisladamente por cada uno de los grupos nosológicos que lo componen. Al menos, el $50 \%$ de los sujetos diagnosticados de trastorno por uso de sustancias presenta otro diagnóstico psiquiátrico y el $30 \%$ de los pacientes con trastorno mental tienen historia de consumo de sustancias (1). La prevalencia-vida de

\section{ABSTRACT}

This study determines the prevalence of substance abuse disorders, patterns of use and specific sociodemographic and clinical profiles, among outpatients in treatment for schizophrenia. Our sample comprised 72 subjects. The Substance Detection Schedule (World health Organization) and the PANSS were used. The current prevalence (previous 12 months) for any substance was $37,5 \%$ and the lifetime prevalence was $54,2 \%$, and they did not differ significantly between the most of the sociodemographic or clinical groups. We observed a link between substance abuse and: male gender, unemployment, legal problems and low compliance with treatments were associated to substance abuse.

Key Words: Schizophrenia, substance abuse, dual diagnosis.

trastornos por uso de sustancias en esquizofrénicos tratados oscila entre el 20 y el $40 \%(1,2)$; se estima que en esta enfermedad el riesgo relativo de padecer un trastorno por uso de sustancias se incrementa 4,6 veces (6,2 si se excluye el alcohol) (2).

Para tratar de explicar la naturaleza del trastorno dual, la asociación entre la esquizofrenia y el abuso de sustancias se han postulado teorías que han servido de base a distintos modelos: la teoría etiológica o de la vulnerabilidad según el la cual en que la psicosis estaría desencadenada por determinadas sustancias psicotropas; la que postula la existencia de una disfunción dopaminérgica común, la de la automedicación, que 
propone la utilización de sustancias con la finalidad de contrarrestar los síntomas psicóticos, la de promoción de la socialización y, finalmente, la que considera que se trata de dos trastornos independientes que coexisten simultáneamente en el mismo sujeto.

En general, se acepta que el trastorno dual ocurre con más frecuencia en varones jóvenes $(5,6,7,8)$, con antecedentes familiares de consumo de alcohol y tóxicos (9)), con mejor ajuste premórbido $(7,9)$ y con un nivel igual o menor de la intensidad de la psicopatología $(5,9,10,11,12)$. También se ha asociado con frecuencia superior a la normal con la aparición de: conducta violenta y destructiva $(13,14)$, marginalidad, funcionamiento sociofamiliar deficiente, mala adherencia al tratamiento $(15,16)$ y refractariedad al tratamiento neuroléptico (17). El número de hospitalizaciones psiquiátricas se observa incrementado en algunos estudios $(6,8)$, mientras que en otros resulta igual o incluso menor $(5,7,9,18)$. Algunos autores han observado la existencia de asociación entre el abuso de sustancias y mayor presencia de síntomas positivos $(19,20)$, alucinaciones $(21,22,23)$, delirios $(22,24)$ y desorganización del lenguaje $(16,22)$, mientras que otros no encuentran relación alguna $(7,11,25,26)$. Los síntomas negativos no suelen ser prominentes en los pacientes con trastorno dual $(27,28)$; pero sí lo son los síntomas depresivos y las conductas suicidas (4, $5,16,29,30)$. En cuanto al impacto que las diferentes sustancias pueden tener sobre el curso del trastorno mental se advierten diferencias, así mientras que la cocaína exacerba los síntomas psicóticos (31), no se ha evidenciado que el consumo de alcohol y cannabis produzca una intensificación de la sintomatología (32).

El presente estudio se plantea como objetivo conocer la prevalencia de consumo de las sustancias psicotropas de uso más frecuente en nuestro medio, en una muestra de pacientes esquizofrénicos tratados, describir los patrones de consumo más habituales y estudiar la presencia de perfiles sociodemográficos y clínicos asociados con el abuso de sustancias.

\section{METODOLOGÍA}

El trabajo se diseñó como un estudio descriptivo de corte transversal. La muestra está compuesta por los pacientes con diagnóstico clínico de psicosis esquizofrénica (criterios CIE-10), con edades comprendidas entre 18 y 65 años, que estaban en contacto con los dispositivos ambulatorios del área VII de los Servicios de Salud del Principado de Asturias y que aceptaron participar en el estudio durante los tres meses establecidos para la recogida de los datos. Los pacientes con trastorno mental orgánico comórbido y aquellos cuyo estado físico y/o psíquico desaconsejaba la realización del estudio fueron excluidos.
La recogida de datos se realizó mediante: un protocolo diseñado "ad hoc" que incluía: datos de filiación y sociodemográficos ( fecha de nacimiento, edad, sexo, estado civil, convivencia, nivel de estudios, situación y actividad laboral), datos clínicos de la enfermedad psicótica (edad de inicio , número de ingresos, años de evolución, forma sindrómica ) tratamiento y adherencia a éste (se consideró adherencia al tratamiento correcta si existía una toma adecuada de la medicación prescrita -autoinforme del paciente- y asistencia regular a las consultas -dato de historia clínica), contactos con el sistema judicial e historia de consumo . La evaluación de los síntomas del trastorno psicótico se realizó mediante la PANSS $(33,34)$, utilizando el sistema inclusivo para la clasificación de los sujetos. La historia del consumo se obtuvo mediante la administración del Cuestionario de Detección del Consumo de Alcohol y Tóxicos de la OMS (36), instrumento que permite evaluar los tóxicos consumidos durante el año y mes previo y a lo largo de la vida, basándose en la información obtenida del paciente y familiares; las sustancias estudiadas fueron: cannabis, cocaína, anfetaminas y otros estimulantes, alucinógenos, cola, aerosoles y otras sustancias volátiles, tranquilizantes (benzodiacepinas), sedantes (barbitúricos), opio, heroína, otros opiáceos y éxtasis. Las limitaciones de este instrumento son las mismas que las de otros que dependen únicamente de la información aportada por el paciente. Para minimizar este problema se trató, cuando fue posible, de que el paciente fuese acompañado de un familiar que confirmase la información. Se valoró, así mismo, la existencia de abuso o dependencia actual o en el pasado para las distintas sustancias estudiadas, siguiendo criterios CIE-10.

El análisis estadístico se realizó utilizando el paquete SPSS, utilizando el estadístico chi cuadrado para evaluar la asociación entre las variables cualitativas y la prueba de $\mathrm{t}$ de student para la comparación de medias de variables cuantitativas.

\section{RESULTADOS}

Las características sociodemográficas y clínicas de la muestra estudiada se presentan en las tablas 1, 2 y 3. El perfil del paciente estudiado se corresponde con un varón, con una edad media de 35,6 (DE=10), que vive con su familia de origen, soltero y con una incapacidad laboral transitoria o permanente. La edad media de inicio de la esquizofrenia fue de 24 años $(D E=9,4)$ la duración media de 13,5 años $(D E=13,8)$ y la media de ingresos psiquiátricos de 3,4 (DE=3,2). No se encontraron diferencias significativas de género en las variables citadas previamente (tabla 1). 
Tabla 1. Características sociodemográficas de la muestra

\begin{tabular}{|c|c|c|c|c|c|}
\hline & \multicolumn{2}{|c|}{$\begin{array}{l}\text { Trastorno por uso de } \\
\text { sustancias }(n=39)\end{array}$} & \multicolumn{2}{|c|}{$\begin{array}{l}\text { No trastorno por uso de } \\
\text { sustancias }(n=33)\end{array}$} & \multirow[b]{2}{*}{$\mathbf{p}$} \\
\hline & $\mathbf{n}$ & $\%$ & $\mathbf{N}$ & $\%$ & \\
\hline \multicolumn{6}{|l|}{ Genero } \\
\hline Hombre & 34 & 87,2 & 18 & 54,5 & $<0,01$ \\
\hline Mujer & 5 & 12,8 & 15 & 45,5 & \\
\hline Total & 39 & 100,0 & 33 & 100,0 & \\
\hline \multicolumn{6}{|l|}{ Estado civil } \\
\hline Soltero & 30 & 76,9 & 22 & 66,7 & ns \\
\hline Casado & 6 & 15,4 & 6 & 18,2 & \\
\hline Otros & 3 & 7,7 & 5 & 15,2 & \\
\hline Total & 39 & 100,0 & 33 & 100,0 & \\
\hline \multicolumn{6}{|l|}{ Convivencia } \\
\hline Familia propia & 6 & 15,4 & 8 & 24,2 & ns \\
\hline Familia de origen & 32 & 82,1 & 21 & 63,6 & \\
\hline Solo & 1 & 2,6 & 4 & 12,1 & \\
\hline Total & 39 & 100,0 & 33 & 100,0 & \\
\hline \multicolumn{6}{|l|}{ Sit laboral } \\
\hline Activo & 2 & 5,1 & 7 & 21,2 & $<0,05$ \\
\hline Parado & 6 & 15,4 & 4 & 12,1 & \\
\hline Incapacidad & 31 & 79,5 & 22 & 66,7 & \\
\hline Total & 39 & 100,0 & 33 & 100,0 & \\
\hline \multicolumn{6}{|c|}{ Contactos con el sistema iudicial } \\
\hline $\mathrm{Si}$ & 10 & 25,6 & 2 & 6,1 & $<0,05$ \\
\hline No & 29 & 74,4 & 31 & 93,9 & \\
\hline Total & 39 & 100,0 & 33 & 100,0 & \\
\hline
\end{tabular}

Tabla 2. Características clínicas de la muestra

\begin{tabular}{|c|c|c|c|c|c|}
\hline & \multicolumn{2}{|c|}{$\begin{array}{l}\text { Trastorno por uso de } \\
\text { sustancias }(n=39)\end{array}$} & \multicolumn{2}{|c|}{$\begin{array}{l}\text { No trastorno por uso de } \\
\text { sustancias }(n=33)\end{array}$} & \multirow[b]{2}{*}{$\mathbf{p}$} \\
\hline & $\mathbf{n}$ & $\%$ & $\mathbf{n}$ & $\%$ & \\
\hline \multicolumn{6}{|c|}{ Subtipo de esquizofrenia } \\
\hline Paranoide & 32 & 82,1 & 22 & 66,7 & ns \\
\hline Otras & 7 & 17,9 & 11 & 33,3 & \\
\hline Total & 39 & 100,0 & 33 & 100,0 & \\
\hline \multicolumn{6}{|l|}{ Subtipo PANSS } \\
\hline Negativo & 18 & 46,2 & 16 & 48,5 & ns \\
\hline Positivo & 1 & 2,6 & 1 & 3,0 & \\
\hline Mixto & 20 & 51,3 & 16 & 48,5 & \\
\hline Total & 39 & 100,0 & 33 & 100,0 & \\
\hline \multicolumn{6}{|c|}{ Adherencia al tratamiento } \\
\hline Buena & 28 & 71,8 & 33 & 100,0 & $<0,01$ \\
\hline Irregular o mala & 11 & 28,2 & 0 & 0,0 & \\
\hline Total & 39 & 100,0 & 33 & 100,0 & \\
\hline
\end{tabular}


Tabla 3. Media y desviación estándar (DE) de algunas características clínicas de la muestra

\begin{tabular}{|c|c|c|c|c|c|}
\hline & \multicolumn{2}{|c|}{$\begin{array}{l}\text { Trastorno por uso } \\
\text { de sustancias }(n=39)\end{array}$} & \multicolumn{2}{|c|}{$\begin{array}{l}\text { No trastorno por uso } \\
\text { de sustancias }(n=33)\end{array}$} & \multirow[b]{2}{*}{$\mathbf{p}$} \\
\hline & media & DE & media & DE & \\
\hline Duración media de la psicosis & 13,2 & 16,7 & 13,9 & 9,4 & ns \\
\hline Edad media de inicio de la psicosis & 24,2 & 11,4 & 24,0 & 6,6 & ns \\
\hline Número medio de ingresos & 3,7 & 11,4 & 3,0 & 3,3 & ns \\
\hline $\begin{array}{l}\text { Número medio de ingresos involuntarios } \\
\text { PANSS }\end{array}$ & 1,6 & 2,1 & 1,2 & 2,6 & ns \\
\hline Positiva & 13,4 & 5,7 & 11,8 & 3,8 & ns \\
\hline Negativa & 19,4 & 7,7 & 20,8 & 8,0 & ns \\
\hline Total & 63,4 & 17,1 & 64,7 & 17,1 & ns \\
\hline
\end{tabular}

Durante el año previo a la evaluación, 27 pacientes $(37,5 \%)$ presentaron consumos regulares con patrón desadaptativo y $39(54,2 \%)$ cumplían criterios para trastorno por uso de sustancias ( abuso y dependencia) actual o en el pasado. En el 76,9\% de los pacientes en los que se objetivó trastorno por uso de sustancias, éste se había iniciado previamente al comienzo de la enfermedad esquizofrénica. En la tabla 4 se muestran los resultados de las prevalencia actual y a lo largo de la vida para las distintas sustancias estudiadas.

Entre los individuos con consumo activo durante el último año $(n=27)$ se encuentra que el $55,5 \%$ consumía dos o más tóxicos. Entre los patrones de uso de sustancias identificados en estos 27 pacientes, los más frecuentes fueron: consumo aislado de alcohol en nueve casos $(33,3 \%)$, consumo aislado de cannabis en tres casos $(11,1 \%)$, consumo conjunto de alcohol y cannabis en cinco casos $(18,5 \%)$ y consumo de tres o más sustancias en 7 pacientes (25,9\%).

En el análisis de los datos se observó que la presencia de abuso o dependencia de sustancias se asociaba de manera significativa con algunas características per- sonales; así, los pacientes con trastorno dual eran habitualmente hombres, estaban más frecuentemente en situación de incapacidad laboral, tenían peor adherencia al tratamiento y contacto con el sistema judicial con mayor frecuencia. No se observaron diferencias significativas en la prevalencia de trastorno por uso de sustancias en relación con el resto de variables sociodemográficas, clínicas o de antecedentes familiares. Tampoco se observaron diferencias significativas cuando se compararon las puntuaciones promedio en la PANSS y subescalas positiva y negativa y la presencia de trastorno por uso de sustancias.

\section{DISCUSIÓN}

La prevalencia de los trastornos por uso de sustancias en la población estudiada es alta $(54,2 \%)$ y comparable a la de otros estudios que muestran tasas que oscilan entre el 20 y el $40 \%$ (4), justificándose la variabilidad por diferencias en los hábitos diagnósticos y/o diferencias en los tipos de poblaciones y características de los pacientes estudiados.

Tabla 4. Prevalencia actual y a lo largo de la vida de trastorno por uso de las sustancias sustancias estudiadas $(n=32)$.

\begin{tabular}{|c|c|c|c|c|c|c|c|c|}
\hline & \multicolumn{4}{|c|}{ Prevalencia (toda la vida previa) } & \multicolumn{4}{|c|}{ Prevalencia puntual (actual) } \\
\hline & \multicolumn{2}{|c|}{ Abuso } & \multicolumn{2}{|c|}{ Dependencia } & \multicolumn{2}{|c|}{ Abuso } & \multicolumn{2}{|c|}{ Dependencia } \\
\hline & $\mathbf{n}$ & tasa & $\mathbf{N}$ & tasa & $\mathbf{n}$ & tasa & $\mathbf{n}$ & tasa \\
\hline Alcohol & 34 & 47,2 & 3 & 4,2 & 19 & 26,4 & 1 & 1,4 \\
\hline Cannabis & 26 & 36,1 & 2 & 2,8 & 13 & 18,1 & 2 & 2,8 \\
\hline Anfetamina & 15 & 20,8 & 0 & 0,0 & 4 & 5,6 & 0 & 0,0 \\
\hline Cocaína & 17 & 23,6 & 0 & 0,0 & 5 & 6,9 & 0 & 0,0 \\
\hline Heroína & 4 & 5,6 & 12 & 16,7 & 1 & 1,4 & 6 & 8,3 \\
\hline LSD & 11 & 15,3 & 0 & 0,0 & 2 & 2,8 & 0 & 0,0 \\
\hline Extasis & 8 & 11,1 & 0 & 0,0 & 1 & 1,4 & 0 & 0,0 \\
\hline Benzodiacepinas & 6 & 8,3 & 4 & 5,6 & 1 & 1,4 & 4 & 5,6 \\
\hline
\end{tabular}


Los primeros estudios realizados en el campo de la patología dual, señalaban que los esquizofrénicos consumían más drogas estimulantes y alucinógenas que la población general o que grupos de pacientes con otros diagnósticos $(38,39)$. Este consumo selectivo sirvió como base para formular la hipótesis de la automedicación (40). El presente estudio, al igual que otros más recientes (5), señala al alcohol como la sustancia empleada con más frecuencia, seguida del cannabis, pauta compartida con la población general (41). Cabe considerar por tanto, que la elección de la sustancia adictiva estaría condicionada por factores sociodemográficos, accesibilidad y coste más que por la presencia de un determinado perfil de síntomas (39, $42,43)$. En todas las sustancias, las tasas de prevalencia del grupo estudiado fueron superiores a las encontradas en los estudios que se han realizado en población general y en poblaciones con otras enfermedades mentales $(2,39,41)$. Como elemento diferencial, cabe reseñar que la prevalencia del trastorno por uso de opiáceos, que en otros estudios presenta una tasa baja, fue el que resultó mas alto al ser comparado con las cifras de prevalencia en población general (41).

La mayor frecuencia de uso de sustancias en los pacientes esquizofrénicos se explicaría en virtud de la existencia, de modo frecuente y persistente, de muchos de los ya conocidos factores de riesgo para presentar una conducta adictiva, a saber: falta de habilidades sociales, alteraciones cognitivas, pobre autoestima, escaso autocontrol, capacidad de afrontamiento restringida y nula o escasa red social de apoyo (44). En los pacientes que, antes del inicio de la enfermedad, hayan tenido contacto con algún tóxico (hecho éste que podría estar influido por factores sociodemográficos, de accesibilidad y disponibilidad y por el propio ajuste premórbido), en el momento de instauración de la psicosis tratarían de aliviar el distress que esta produce con un reinicio o mantenimiento del consumo, produciéndose secundariamente una escalada en el consumo hacia niveles patológicos (7).

Nuestros resultados coinciden con los de la literatura revisada, tanto en la alta prevalencia del policonsumo $(3,5,39,43,45,46)$, como en los patrones de consumo encontrados, que son coincidentes con los identificados por autores como Cuffel, el cual señala en uno de sus estudios que el $54 \%$ de los pacientes eran no consumidores, un $31 \%$ sufría trastorno por uso de alcohol y cannabis y el resto (14\%) eran consumidores de múltiples sustancias.

En cuanto a las características asociadas al consumo ya Drake (16) había postulado el peor ajuste social, manifestado en su estudio por la incapacidad laboral o el mayor número de contactos con el sistema judicial. Sin embargo, no hemos encontrado asociaciones significativas entre la presencia de un trastorno por uso de sustancias y una expresión diferencial de la sintomatología.

Todos los pacientes con una baja adherencia al tratamiento presentaban un trastorno por uso de sustancias, con consumo activo y regular. Este dato ya señalado en otros trabajos $(15,16)$ podría traducirse en la práctica clínica diaria en una mayor dificultad de manejo ambulatorio, aunque curiosamente no parece traducirse en un mayor número de hospitalizaciones en nuestro caso. En este sentido, y teniendo en cuenta la aparente ausencia de repercusión tanto en el perfil de los síntomas como en el número de hospitalizaciones, y en la misma línea propuesta por autores como Dixon (9) se podría hipotetizar a favor de la existencia de formas menos severas de la enfermedad para alguno de los pacientes con trastorno por uso de sustancias.

\section{BIBLIOGRAFÍA}

(1) Cuffel BJ. Comorbid substance use disorders: prevalence, patterns of use, and course. New Dir Ment Health Serv 1996; 70: 93-105.

(2) Mueser KT, Bellack AS, Blanchard JJ. Comorbidity on schizophrenia and substance abuse: implications for treatment. Journal of Consulting and Clinical Psycology 1992; 60: 845-856.

(3) Cohen T, Jackson A.M. Dual Diagnosis: substance abuse and psychiatric illness. Philadelphia: Hanley \& Belfus Inc 1996. p.129.

(4) Regier DA, Farmer ME, Rae DS, Locke BZ, Keith SJ, Judd LL, Goodwin FK. Comorbidity of mental disorders with alcohol and other drug abuse. JAMA 1990; 264: 25112518.

(5) Schneier FR, Siris SG. A review of psychoactive substance use and abuse in schizophrenia: patterns of drug choice. J Nerv Ment Dis 1987; 175: 641-652.

(6) El-Guebali N, Hodgins DC. Schizophrenia and substance abuse: prevalence issues. Can J Psychiatry 1992; 37 : 704-710.

(7) Ardnt S, Tyrrell G, Flaum M, Andreasen NC. Comorbidity of substance abuse and schizophrenia. Psychol Med 1992; 22: 379-388.

(8) Drake RE, Osher FC, Noordsy DL. Diagnosis of alcohol use disorders in schizophrenia. Schizophr Bull 1990;16: 57-67.

(9) Dixon L, Haas G, Weiden PJ, Sweeney J, Frances AJ. Drug abuse in schizophrenic patients: clinical correlates and reasons for use. Am J Psychiatry 1991;148: 224.230.

(10) Ritzler BA, Strauss JS, Vanord A, Kokes RF . Prognostic implications of various drinking patterns in psychiatric patients. Am J Psychiatry 1977; 134: 546-549.

(11) D'mello DA, Boltz MK, Msibi B . Relationship between concurrent substance abuse in psychiatric patients and 
neuroleptic dosage. Am J Drug Alcohol Abuse 1995; 21: $257-265$

(12) Caton CL, Gralnick A, Bender S, Simon R. Young chronic patients and substance abuse. Hosp Community Psychiatry 1989; 40: 1037-1040.

(13) Smith J, Hucker S. Schizophrenia and substance abuse. Br J Psychiatry 1994; 165: 13-21.

(14) Cuffel BJ, Sumway M, Choulijan TL, MacDonald T . A longitudinal study of substance use and community violence in schizophrenia. J Nerv Ment Dis 1994; 182: 704-708.

(15) DeQuardo JR, Carpenter CF, Tandon R. Patterns of substance abuse in schizophrenia: nature and significance. J Psychiatr Res 1994; 28: 267-275.

(16) Drake RE, Wallach MA. Substance abuse among the chronic mentally ill. Hosp Community Psychiatry 1989; 40: 1041-1046.

(17) Bowers MB, Mazure M, Craig J, Jatlow P. Psychotogenic drug use and neuroleptic response. Schizophr Bull 1990; 16: 81-85.

(18) Verdoux H, Mury M, Besancon G, Bourgeois M. Comparative study of substance dependence comorbidity in bipolar, schizophrenic and schizoaffective disorders. Encephale 1996;22: 95-101.

(19) Allebeck P, Adamsson C, Engström A, Rydberg U. Cannabis and schizophrenia: A longitudinal study of cases trated in Stockholm County. Acta Psychiatr Scand 1993; 88: 21-24.

(20) Knudsen P, Wilmar R. Cannabis and neuroleptics agents in schizophrenia. Acta Psychatr Scan 1984; 69: 162174.

(21) Sokolski KN, Cummings JL, Abrams BI, DeMet EM, Katz LS, Costa JF. Effects of substance abuse on hallucination rates and treatments responses in chronic psychiatric patients. J Clin Psychiatry 1994; 55: 380387.

(22) Cleghorn JM, Kaplan RD, Szechtman B, Szechtman H, Brown GM, Franco S. Substance abuse and shizophrenia: effects on symptoms but not on neurocognitive function. J Clin Psychiatry 1991; 52: 26-30.

(23) Barbee JG, Clark PD, Crapanzano MS, Heintz GC, Kehoe CE. Alcohol and substance abuse among schizophrenic patients presenting to an emergency psychiatric service. J Nerv Ment Dis 1989; 177: 400407.

(24) Negrete JC, Knapp WP, Douglas DE . Cannabis affects de severity of schizophrenic symptoms: results of a clinical survey. Psycol Med 1986; 16: 515-520.

(25) Shaner A, Khalsa ME, Roberts L, Wilkins J, Anglin D, Hsieh S. Unrecognised cocaine use among schizophrenic patients. Am J Psychiatry 1993; 150: 758-762.

(26) Sevy S, Kay SR, Opler LA, Van Praag HM. Significance of cocaine history in schizophrenia. J Nerv Ment Dis 1993; 178: 642-648.

(27) Dixon L, Haas G, Weiden PJ, Sweeney J, Frances AJ. Acute effects of drug abuse in schizophrenic patients: clinical observations and patient's self reports. Schizophr Bull 1990; 16: 69-79.
(28) Andreasen NC . Negative symptoms in schizophrenia: definition and reliability. Arch Gen Psychiatry 1982; 39: 784-788.

(29) Soyka M, Albus M, Kathmann N, Finelli A, Hofstetter S, Holzbach R, Immler B, Sand P. Prevalence of alcohol and drug use in schizophrenic inpatients. Eur Arch Psychiatry Clin Neurosci 1993; 242: 362-372.

(30) Brunette MF, Mueser KT, Xie H, Drake RE. Relatioships between symptoms of schizophrenia and substance abuse. J Nerv Ment Dis 1997; 185: 13-20.

(31) Shaner A, Eckman TA, Roberts LJ, Wilkins JN, Tucker D, Tsuang JW, Mintz J. Disability income, cocaine use and repeated hospitalization among schizophrenic cocaine abusers: a goverment sponsored revolving door?. New Engl J Med 1995; 333: 777-783.

(32) Hamera E, Schneider JK, Deviney S. Alcohol, cannabis, nicotine and caffeine use and symptom distress in schizophrenia. J Nerv Men Dis 1995; 183: 559-565.

(33) Kay SR, Opler LA (1987). The positive-negative dimension in schizophrenia: its validity and significance. Psychiatr Dev, 1987; 2:79.

(34) Peralta V, Cuesta MJ. La validación de la escala de síndromes positivo y negativo (PANSS).Actas LusoEsp Neurol Psiquiatr Cienc afines 1994; 22: 171-177.

(35) Montgomery SA, Asberg M. A new depression scale designed to be sensitive to change. Brit J Psychiat 1979; 134: 382-389.

(36) Smart HG, Hughes PH, Johnston LD. Methodologie pour des enquetes sur l'usage des drogues chez les etudiants. Publiciten Offset, $n^{\circ} 50$, Geneve, OMS 1980.

(37) CIE-10 .Trastornos Mentales y del Comportamiento. Versión multiaxial para adultos. OMS, Madrid; 1996. p.155-162

(38) Schneier FR, Siris SG. A review of psychoactive substance use and abuse in schizophrenia: patterns of drug choice. J Nerv Ment Dis 1987; 175: 641-652.

(39) Mueser KT, Yarnold PR, Levinson DF, Singh H, Bellack AS, Kee K, Morrison RL, Yadalan KG. Prevalence of substance abuse in schizophrenia: demographic and clinical correlates. Schizophr Bull 1990; 16: 31-52

(40) Khantzian EJ. The self medication hipothesis of addictive disorders: focus on heroin and cocaine dependence. Am J Psychiatry 1985; 142: 1259-1264.

(41) Plan Nacional sobre drogas. Memoria 1996. Madrid: ed. Ministerio del Interior, 1997. p. 22-23

(42) Lambert M, Haasen C, Mass R, Krausz M. Consumption patterns and motivation for use of addictive drugs in schizophrenic patients. Psychiatr Prax 1997; 24: 185-189.

(43) Baigent M, Holme G, Hafner RJ . Self reports of the interaction between substance abuse and schizophrenia. Aust N Z J Psychiatry 1995; 29: 69-74.

(44) Donovan DM. Assesment of addictive behaviors: implications of an emerging biopsychosocial model. En Assesment of addictive behaviors. New York: Ed Donovan DM, Marlatt GA.1998. 
(45) Test MA, Yallisch LS, Allness DJ, Ripp K. Substance abuse in young adults with schizophrenic disorders. Shizophr Bull 1989; 15: 465-476.
(46) Modestín J, Nussbaumer C, Angst K, Scheidegger P, Hell D. Use of potentially abusive psychotropic substances in psychiatric inpatients. Eur Arch Psychiatry Clin Neurosci 1997; 3: 146-153. 
